УДК 330.142 .211

И. М. Щадов

Иркутский национальный исследовательский технический университет,

г. Иркутск, Российская Федерация

В. Ю. Конюхов

Иркутский национальный исследовательский технический университет,

2. Иркутск, Российская Федерация

А. В. Чемезов

ООО «Норильскникельрелонт», 2. Норильск, Российская Федерация

Т. С. Беляевская

Национальный исследовательский университет

«Высшая школа эконолики»,

2. Москва, Российская Федерация

\title{
ИНВЕСТИРОВАНИЕ ИМПОРТОЗАМЕЩЕНИЯ НА ПРЕДПРИЯТИЯХ МЕТАЛЛУРГИЧЕСКОЙ ОТРАСЛИ
}

АНнотАция. В данной статье проводится обзор текущей ситуации в отечественной металлургической отрасли посредством анализа рыночной ситуации и состояния технологического оборудования. Рассматривается роль инвестирования в повышение эффективности работы крупного промышленного предприятия в условиях нестабильной экономической ситуации в стране. В текущей ситуации высоких курсов иностранных валют, а также запрещенного ввоза импортного оборудования на территорию РФ, предприятия страны начинают поиск аналогов импортного оборудования на отечественном рынке. На примере одного из подразделений ПАО «ГМК «Норильский Никель» освещен процесс внедрения на производство отечественного расходного материала взамен импортного, а также приведены финансовые, организационные и экономические результаты от проведенных мероприятий по состоянию на конец 2016 и 2017 гг. при условии, что объем работ останется на прежнем уровне. кЛючЕВЫЕ словА. Импортозамещение; инвестирование; металлургическая отрасль; эффективность инвестиционного проекта; технологическое оборудование; структура затрат, срок окупаемости.

ИНФОРМАЦИЯ О СТАТЬЕ. Дата поступления 10 октября 2016 г.; дата принятия к печати 12 ноября 2016 г.; дата онлайн-размещения 30 ноября 2016 г.

\author{
I. M. Schadov \\ Irkutsk National Research Technical University, \\ Irkutsk, Russian Federation \\ V. Yu. Konukhov \\ Irkutsk National Research Technical University, \\ Irkutsk, Russian Federation \\ A. V. Chemezov \\ Norilsknickelremont, \\ Norilsk, Russian Federation \\ T. S. Beliaevskaia \\ Higher School of Economics - National Research University, \\ Moscow, Russian Federation
}

\section{INVESTING IMPORT SUBSTITUTION AT METALLURGIC ENTERPRISES}

ABSTRACT. This article provides an overview of the current situation in the national metallurgical industry through analyzing the market situation and the state of technological equipment. It considers the role of investments in improving performance

\section{Baikal Research Journal}


efficiency of a large industrial enterprise in terms of unstable economic situation in the country. In the current situation of high exchange rates of foreign currencies, as well as the prohibited entry of imported equipment to the territory of the Russian Federation, enterprises of the country are beginning to search for imported equipment analogues in the domestic market. Using the example of one of the divisions of PJSC "MMC Norilsk Nickel» the article highlights the implementation process for the production of domestic consumables to replace the imported ones, as well as presents financial, organizational and economic results of the activities undertaken by the end of 2016 and 2017, provided that the amount of work will remain at the same level. KEYWORDS. Import substitution; investment; metallurgical industry; the efficiency of investment project; technological equipment; cost structure; payback period.

ARTICLE INFO. Received October 10, 2016; accepted November 12, 2016; available online November 30, 2016.

Металлургия, традиционно являясь базовой отраслью промышленности, играет ключевую роль в экономике России как один из основных поставщиков инвестиций в основной капитал и индикатор социально-экономического развития. В настоящее время наличие негативных тенденций в деятельности предприятий отечественной металлургической промышленности обусловлено рядом факторов, затрудняющих развитие отрасли. С одной стороны, это внешние факторы, определяющие условия функционирования металлургических предприятий, к основным из которых относятся:

1. Низкий уровень спроса на отечественную металлопродукцию на внутреннем рынке, прежде всего со стороны металлообрабатывающих предприятий и машиностроительной отрасли.

2. Отсутствие спроса на российскую металлопродукцию высоких переделов на внешних рынках сбыта.

3. Резкое усиление экспансии Китая и других азиатских стран на мировых рынках металлопродукции; падение мировых цен на металлургическую продукцию, и прежде всего на цветные металлы.

4. Завышенные процентные ставки по кредитам.

5. Отсутствие комплексной государственной программы по поддержке отрасли [1].

Кроме внешних факторов, развитие металлургической промышленности России сдерживается и рядом внутриотраслевых факторов [2], а именно:

1. Высокий уровень износа основных промышленно-производственных фондов и значительное технологическое отставание на ряде предприятий.

2. Неконкурентоспособность отдельных видов используемого рудного сырья и ограниченность ряда видов сырьевых ресурсов.

3. Дефицит некоторых видов металлопродукции.

4. Неразвитость сети предприятий малого и среднего бизнеса, производящих широкую номенклатуру металлоизделий в соответствии с требованиями рынка металлопродукции.

5. Повышенные, по сравнению с зарубежными предприятиями-аналогами, удельные расходы сырья, материальных и энергоресурсов в натуральном выражении на производство однотипных видов металлопродукции.

6. Низкий уровень производительности труда.

7. Проблемы, связанные с охраной окружающей среды на ряде металлургических производств, обуславливающие сверхнормативные выбросы вредных веществ в водные бассейны и атмосферу.

8. Низкая восприимчивость предприятий к внедрению инноваций - прежде всего, отечественных.

\section{Baikal Research Journal}


Таким образом, формирование инновационной политики металлургических предприятий, адекватной современным экономическим условиям, является важнейшей задачей достижения конкурентных преимуществ в долгосрочной перспективе и повышения эффективности промышленного производства. Необходимость осуществления модернизации является следствием выше указанных причин, сдерживающих развитие металлургической промышленности [3].

Стоит отметить, что инвестиции являются двигателем развития не только малого бизнеса, но и крупных промышленных предприятий. В связи с тем, что 1990-2000-е гг. вложения в работу оборудования промышленных предприятий были минимальными, на сегодняшний день возникла ситуация снижения рентабельности многих производств. В свою очередь, инвестирование в промышленные предприятия может оказать положительное влияние на рост его эффективности [4].

В современной экономической ситуации привлечь внимание инвесторов становится все сложнее: инвестиционный рейтинг страны в кризисных условиях не привлекателен, а на федеральном уровне наибольшее предпочтение отечественные инвесторы отдают регионам с наиболее развитой инфраструктурой (Москва и Московская область, Санкт-Петербург, Калининградская область) [5].

Приоритетными отраслями инвестирования по-прежнему являются добыча и переработка полезных ископаемых, металлургия, строительство, крупное производство, инновации.

При оценке эффективности инвестиционного проекта степень точности и достоверности финансовой информации повышается по мере развития жизненного цикла. На начальном этапе любого проекта целесообразно проводить его экспресс-оценку. Особенно важно оценить потенциал инвестирования на этапе формирования идеи и разработки предварительного технико-экономического обоснования (ТЭО) или бизнес - плана проекта. Экспресс - методы основаны на использовании традиционных инструментов дисконтирования денежных потоков, которые применимы для отсева экономически неэффективных из большого числа проектов, рассматриваемых инвестором, например, в случае конкурса или реализации целевой программы. В этом случае проводится элементарная сравнительная критериальная оценка показателей рассматриваемого проекта с приоритетами для инвестора. Для более глубокого и детального анализа и разработки прогноза денежных потоков любого проекта, как автор, так и инвестор могут использовать инструментальные средства инвестиционного проектирования, упрощающие и ускоряющие работу, например, Prime Expert или Project Expert. Если автор проекта будет руководствоваться информацией, касающейся прогнозов в рассматриваемой отрасли, сможет найти исходные данные, применимые при затратно-восстановительном методе, то предварительный финансовый расчет проекта будет адекватным требованиям инвестора к дисконтированным показателям [6].

В соответствие с планами по повышению эффективности производства, ПАО «ГМК «Норильский Никель» был произведен поиск технических решений по снижению трудоемкости производственных процессов, увеличению производительности и повышению уровня промышленной безопасности [7]. Одной из площадок, на которых была произведена работа по повышению эффективности производства, стал цех по изготовлению и ремонту пылевентиляционных систем предприятий цветной металлургии (входит в состав ПО «Норильскремонт» ООО «Норильскникельремонт»). Результатом данного поиска стал выбор оборудования компании Spiro International S.A. (Швейцария), имеющего сертификат соответствия техническим регламентам РФ.

\section{Baikal Research Journal}

электронный научный журнал Байкальского государственного университета 
Оборудование приобретено с целью увеличения качества и производительности работ по раскрою листового металла при выполнении комплекса работ по изготовлению и ремонту пылевентиляционных систем в цехе ПО «Норильскремонт» [8].

Установка плазменной резки Spiro Plasmacutter Cutlass (далее - Установка) применяется для раскроя заготовок из листового металла толщиной до 12 мм (включая нержавеющие стали) при изготовлении фасонных частей круглых, прямоугольных и овальных воздуховодов. Поставлена со специальным программным обеспечением для вентиляционной промышленности с полной библиотекой трехмерных параметрических моделей фасонных деталей систем воздуховодов, изоляции и дымоходов.

Установка укомплектована фильтровальным вентиляционным агрегатом полного цикла фильтрования воздуха с функцией пневматической самоочистки. Полная масса Установки - 3 т. Размеры рабочего стола позволяют обрабатывать листовой металл стандартных размеров (1500 х 6000 мм). Двухкоординатная система позиционирования режущего блока дает возможность использовать всю площадь рабочего стола и добиться как высокой скорости резки материала (40 м/мин), так и высокой точности (0,2 мм).

Контроль всех параметров и управление Установкой осуществляется промышленным компьютером при помощи сенсорных дисплеев. После ориентации заготовки на поверхности стола, весь процесс раскроя деталей производится в автоматическом режиме, в соответствие с алгоритмом программы [9].

Автоматизированная резка и прожиг листовых материалов осуществляется системой плазменной резки Powermax65 (производства компании HIPERTHERM, США), укомплектованным механизированным резаком $180^{\circ}$. Система позволяет осуществлять резку черных и цветных металлов с высокой скоростью и качеством. Скорость резки низкоуглеродистой стали толщиной 12 мм в полтора раза выше по сравнению с аналогичным показателем для кислородной резки. Превосходное качество резки позволяет сократить время, которое затрачивается на шлифование и подготовку кромок. Для работы системы не применяются горючие газы, используется чистый, сухой, обезжиренный воздух с автоматической системой регулирования давления.

С 1 июня 2015 г. Установка запущена в опытно-промышленную эксплуатацию. По итогам эксплуатации Установки в течение 7 месяцев, 1 января 2016 г. были полностью подтверждены основные технические параметры и эффективность применения системы данного типа, в том числе:

- размеры обрабатываемого листового металла 1500 х 6000 мм;

- толщина обрабатываемого металла до 12 мм;

- точность резки - 0,2 мм;

- скорость резки (с приводом на зубчатые рейки по осям X и Y, макс. 40 м/мин);

- удобство (свободный доступ к рабочей зоне со всех четырех сторон);

- безопасность (датчики прикосновения, на подвижных частях, обеспечивают экстренную остановку машины в случае соприкосновения с оператором, вырезаемой деталью или отходом);

- экология рабочего места (внутренняя вытяжная система, эффективно удаляет продукты горения непосредственно из-под горелки, что при совместной работе с внутрицеховой системой приточно-вытяжной вентиляции обеспечивает безопасность выполнения работ);

- универсальность (программное обеспечение CAMduct 2015 обеспечивает работу с полной библиотекой трехмерных параметрических моделей; осуществляется загрузка чертежей из AutoCAD; выкройки на листе располагаются оптимально).

\section{Baikal Research Journal}


В результате внедрения Установки, из эксплуатации выведены ножницы ЦНТА-10, НД3318 и НГ5222. При односменном режиме работы потребление электроэнергии оборудованием за 7 месяцев до и после внедрения Установки показано в табл. 1.

Таблица 1

Потребление электроэнергии за 7 месяцев

\begin{tabular}{|c|c|c|c|c|c|}
\hline Наименование & $\begin{array}{c}\text { Мощность, } \\
\text { кВт }\end{array}$ & \begin{tabular}{|c} 
Коэффициент \\
использования \\
оборудования \\
\end{tabular} & $\begin{array}{l}\text { Баланс } \\
\text { рабочего } \\
\text { времени }\end{array}$ & $\begin{array}{c}\text { Потребление } \\
\text { электроэнергии, } \\
\text { тыс. кВт · ч }\end{array}$ & $\begin{array}{l}\text { Затраты на } \\
\text { электроэнер- } \\
\text { гию, тыс. p. }\end{array}$ \\
\hline \multicolumn{6}{|c|}{ До внедрения } \\
\hline \multicolumn{6}{|l|}{ Ножницы } \\
\hline ЦНТА-10 & 22 & 0,55 & 1213 & 14,6773 & 14,530 \\
\hline НД3318 & 8,5 & 0,55 & 1213 & 5,6707 & 5,614 \\
\hline НГ5222 & 4,8 & 0,55 & 1213 & 3,2023 & 3,170 \\
\hline Bcero & 35,3 & - & - & 23,5503 & 23,314 \\
\hline \multicolumn{6}{|c|}{ После внедрения } \\
\hline $\begin{array}{l}\text { Установка Spiro } \\
\text { Plasmacutter Cutlass }\end{array}$ & 11,0 & 0,55 & 1213 & 7,3380 & 7,2652 \\
\hline
\end{tabular}

Экономия электроэнергии, в результате вывода из эксплуатации трех единиц ножниц, за 7 месяцев составила 16,212 3 тыс. кВт · ч на сумму 16,048 тыс. р.

За 7 месяцев 2015 г. (с июня по декабрь), производственная программа по изготовлению воздуховодов составила $3758 \mathrm{~m}^{2}$.

После внедрения в производство установки плазменной резки Spiro Plasmacutter Cutlass, производство воздуховодов, увеличилось на $663 \mathrm{~m}^{2}$, за счет дополнительно поступивших заказов ${ }^{1}$.

Производственные показатели, за отчетный период (7 месяцев) составили:

- $21 \%$ - рост объемов производства систем ПВС;

- $40 \%$ - снижение трудозатрат на участке раскроя (сокращение 2 чел. 2426 чел./ч);

- $10 \%$ - снижение количества лома листовых прокатов черных и цветных металлов.

В общей производственной заявке цеха, отношение секций воздуховодов составляет:

- изготавливаемых, с использованием ножниц гильотинных, прямолинейных секций $-80 \%$;

- изготавливаемых, с использованием Установки, криволинейных фасонных частей $-20 \%$.

За период опытно-промышленной эксплуатации Установки (7 месяцев), неисправностей не выявлено, необходимые комплектующие в наличии, расходные материалы и быстро изнашиваемые части внесены в потребность закупа 2016 г.

Дополнительно, с целью подбора качественных аналогов дорогостоящим оригинальным расходным материалам импортного производства (США), проведены сравнительные испытания комплектов сопел для плазменного резака, производства компании Autocut (Россия). Комплектующие показали высокую стойкость, а также качество и заключением комиссии, по результатам опытно-промышленных испытаний, рекомендованы к применению в ПО «Норильскремонт» [10].

Производственная программа участка в 2016 г. предусматривает изготовление воздуховодов из металла толщиной от 1,5 мм до 12 мм в количестве $10500 \mathrm{~m}^{2}$ $\left(190\right.$ т) в год, что составляет около $875 \mathrm{~m}^{2}(15,83$ т) в месяц. При этом производство воздуховодов различных типов за месяц может достигать: $1345 \mathrm{~m}^{2}$ (стальной прокат 1,5 мм); 672 м $^{2}$ (стальной прокат 3 мм); 168 м² $^{2}$ (стальной прокат 12 мм).

\footnotetext{
${ }^{1}$ Экспертные данные специалистов ООО «Норильскникельремонт» (от 12 янв. 2016 г.).
}

\section{Baikal Research Journal}


Производительность Установки в смену составляет от 4 до 8 листов проката (36-72 м², при толщине стального проката 12-1,5 мм), что обеспечит выполнение плана по раскрою прямолинейных и фасонных частей воздуховодов в количестве 792-1584 м² в месяц. В связи с достаточной производительностью, Установка заменит имеющийся парк оборудования на участке прямого и фасонного раскроя.

Экономия электроэнергии, в результате вывода из эксплуатации пяти единиц оборудования, за 2016 г. составит 37,9254 тыс. кВт · ч на сумму 37,55 тыс. р. (табл. 2)

Таблица 2

Планируемый вывод из эксплуатации (в резерв) оборудования в 2016 г.

\begin{tabular}{|c|c|c|c|c|c|}
\hline Наименование & $\begin{array}{c}\text { Мощность, } \\
\text { кВт }\end{array}$ & $\begin{array}{c}\text { Коэффициент } \\
\text { использования } \\
\text { оборудования }\end{array}$ & $\begin{array}{c}\text { Годовой } \\
\text { баланс } \\
\text { рабочего } \\
\text { времени, ч }\end{array}$ & \begin{tabular}{|c|} 
Годовое потре- \\
бление электро- \\
энергии, тыс. \\
кВт $\cdot$ ч \\
\end{tabular} & $\begin{array}{c}\text { Затраты } \\
\text { на электро- } \\
\text { энергию, } \\
\text { тыс. p. } \\
\end{array}$ \\
\hline $\begin{array}{l}\text { Ножницы гильотинные } \\
\text { для резки металла Д }\end{array}$ & 11,00 & 0,25 & 1974 & 5,42850 & 5,37 \\
\hline Ножницы гильотинные & 7,50 & 0,25 & 1974 & 3,70125 & 3,66 \\
\hline $\begin{array}{l}\text { Гильотинные ножницы } \\
\text { МТ-16/20/31-50 }\end{array}$ & 11,00 & 0,25 & 1974 & 5,42850 & 5,37 \\
\hline $\begin{array}{l}\text { Ножницы гильотинные } \\
\text { НА } 3221\end{array}$ & 14,35 & 0,25 & 1974 & 7,08170 & 7,02 \\
\hline $\begin{array}{l}\text { Установка плазмен- } \\
\text { ной резки Мульти- } \\
\text { плаз-15000 }\end{array}$ & 15,00 & 0,55 & 1974 & 16,28550 & 16,13 \\
\hline Всего & 58,85 & - & - & 37,92540 & 37,55 \\
\hline
\end{tabular}

При достаточной численности работников на участке раскроя - один оператор Установки, а также сохранения объемов производства на текущем уровне, сокращение численности составит 4 чел. (слесарь по сборке металлоконструкций) (табл. 3). При утвержденном балансе рабочего времени на 2016 г. равном 1974 ч, экономия трудозатрат составит $1974 \cdot 4=7896$ чел./ч (8 657 тыс. р. при стоимости 1 чел./ч - 1096,4р.).

Таблица 3

Планируемое распределение трудовых ресурсов в 2016 г.

\begin{tabular}{|c|c|c|c|c|c|}
\hline \multirow[t]{3}{*}{ Участок } & \multirow[t]{3}{*}{ Специальность } & \multicolumn{4}{|c|}{ Численность трудовых ресурсов при: } \\
\hline & & \multicolumn{2}{|c|}{$\begin{array}{c}\text { опытно-промышленных } \\
\text { испытаниях }\end{array}$} & \multicolumn{2}{|c|}{ эксплуатации } \\
\hline & & чел. & всего, чел. & чел. & всего, чел. \\
\hline \multirow{2}{*}{\begin{tabular}{|l|} 
Фасонного раскроя \\
Прямого раскроя \\
\end{tabular}} & \multirow{3}{*}{$\begin{array}{l}\text { Слесарь по сборке } \\
\text { металлоконструкций }\end{array}$} & 1 & 1 & 1 & \\
\hline & & 2 & 2 & 1 & 1 \\
\hline \multirow{2}{*}{ Сборки } & & 4 & \multirow{2}{*}{8} & 3 & \multirow{2}{*}{6} \\
\hline & Электросварщик & 4 & & 3 & \\
\hline \multirow{2}{*}{$\begin{array}{l}\text { Заготовки фланцев и } \\
\text { обварки }\end{array}$} & $\begin{array}{l}\text { Слесарь по сборке } \\
\text { металлоконструкций }\end{array}$ & 2 & \multirow[t]{2}{*}{3} & 2 & \multirow[t]{2}{*}{3} \\
\hline & Электросварщик & 1 & & 1 & \\
\hline \multirow{2}{*}{$\begin{array}{l}\text { Антикоррозионной за- } \\
\text { щиты (грунтования) }\end{array}$} & Изолировщик-пленочник & 2 & 2 & 2 & 2 \\
\hline & & & 16 & & 12 \\
\hline
\end{tabular}

Кроме того, планируемая экономия средств, при замене расходных материалов импортного производства (сопла Hipertherm, производства США), на аналоги российского производства (комплектов сопел производства компании Autocut), за год составит 303,6 тыс. p.

\section{Baikal Research Journal}


Итак, затраты на приобретение Установки с доставкой заказчику и проведением пусконаладочных работ составили 9800 тыс. р. Однако, значительное снижение трудозатрат в следующем году и сопутствующее снижение расхода электроэнергии позволят в 2017 г. выйти на положительный финансовый результат.

Таким образом, можно сказать, что инвестирование в основные производственные фонды предприятия позволяет повысить качество продукции, способствует увеличению производительности труда и позволяет привлечь новых заказчиков.

\section{Список использованной литературы}

1. Руйга И. Р. Ключевые проблемы и факторы, сдерживающие развитие отечественной металлургической промышленности / И. Р. Руйга // Современные проблемы науки и образования. - 2014. - № 5. - С. 373 .

2. Руйга И. Р. Особенности инновационного и инвестиционного развития металлургической промышленности Российской Федерации / И. Р. Руйга // Инновационная наука. 2015. - № 8. - C. 58-62.

3. Инвестирование в повышение эффективности сервисного обслуживания технологического оборудования промышленного предприятия / И. М. Щадов, В. Ю. Конюхов, А. В. Чемезов, Т. С. Беляевская // Известия вузов. Инвестиции. Строительство. Недвижимость. - 2016. - № 1 (16). - С. 82-89.

4. Соболев А. С. Оценка экономической привлекательности инвестиционных проектов на стадии разработки идеи / А. С. Соболев // Вестник Иркутского государственного технического университета. - 2015. - № 10. - С. 249-253.

5. The control mechanisms on the performance of the strategic initiatives management: Analysis of critical sales process in a metallurgical business / C. F. Rocha, L. C. Duclos, L. P. da Veiga, C. B. dos Santos, N. A. F. Neves // International Business Management. 2016. - Vol. 10, iss. 4. - P. 357-369. - DOI: 10.3923/ibm.2016.357.369.

6. Razvadovskaya Y. V. Dynamics of metallurgic production in emerging countries / Y. V. Razvadovskaya, I. K. Shevchenko // Asian Social Science. - 2015. - Vol. 11, iss. 19. P. 178-184. - DOI: http://dx.doi.org/10.5539/ass.v11n19p178.

7. Izvekov Y. A. Mathematical modeling and calculation of accuracy and durability of mechanical systems' elements / Y. V. Razvadovskaya, I. K. Shevchenko // World Applied Sciences Journal. - 2014. - Vol. 30, iss. 1. - P. 32-34. - DOI: 10.5829/idosi. wasj.2014.30.01.13990.

8. Kopylov N. I. Problems of arsenic-containing dumps of non-ferrous metallurgy / N. I. Kopylov // Tsvetnye Metally. - 2013. - Iss. 3. - P. 30-35.

9. Halmi M. The investment decision and the economic growth / M. Halmi // Metalurgia International. Special Issue. - 2013. - Vol. 18, iss. 2. - P. 210-213.

10. Lin H.-J. Investing in lead-time variability reduction in a collaborative vendor-buyer supply chain model with stochastic lead time / H.-J. Lin // Computers and Operations Research. - 2016. - Vol. 72. - P. 43-49. - DOI: 10.1016/j.cor.2016.02.002.

\section{References}

1. Ruyga I. R. Key problems and factors restraining the development of national metallurgical industry. Sovremennye problemy nauki i obrazovaniya $=$ Modern Problems of Science and Education, 2014, no. 5, p. 373. (In Russian).

2. Ruyga I. R. Features of innovation and investment development of metallurgic industry of the Russian Federation. Innovatsionnaya nauka = Innovative Science, 2015, no. 8, pp. 58-62. (In Russian).

3. Shchadov I. M., Konyukhov V. Y., Chemezov A. V., Beliaevskaia T. S. Investment in the effectiveness increase of maintenance support of technological equipment of an industrial factory. Izvestiya vuzov. Investitsii. Stroitel'stvo. Nedvizhimost' = Proceedings of Universities. Investments. Construction. Real Estate, 2016, no. 1 (16), pp. 82-89. (In Russian).

4. Sobolev A. S. Investment project economic attractiveness assessment at the stage of idea development. Vestnik Irkutskogo gosudarstvennogo tekhnicheskogo universiteta $=$ Bulletin of Irkutsk State Technical University, 2015, no. 10, pp. 249-253. (In Russian).

\section{Baikal Research Journal}


5. Rocha C. F., Duclos L. C., da Veiga C. P., dos Santos C. B., Neves N. A. F. The control mechanisms on performance of the strategic initiatives management: Analysis of critical sales process in metallurgical business. International Business Management, 2016, vol. 10, iss. 4, pp. 357-369. DOI: 10.3923/ibm.2016.357.369.

6. Razvadovskaya Y. V., Shevchenko I. K. Dynamics of metallurgic production in emerging countries. Asian Social Science, 2015, vol. 11, iss. 19, pp. 178-184. DOI: http://dx.doi. org/10.5539/ass.v11n19p178.

7. Izvekov Y. A., Dubrobsky V. V., Hamutskikh E. Y. Mathematical modeling and calculation of accuracy and durability of mechanical systems' elements. World Applied Sciences Journal, 2014, vol. 30, iss. 1, pp. 32-34. DOI: 10.5829/idosi.wasj.2014.30.01.13990.

8. Kopylov N. I. Problems of arsenic-containing dumps of non-ferrous metallurgy. Tsvetnye Metally, 2013, iss. 3, pp. 30-35.

9. Halmi M. The investment decision and the economic growth. Metalurgia International. Special Issue, 2013, vol. 18, iss. 2, pp. 210-213.

10. Lin H.-J. Investing in lead-time variability reduction in a collaborative vendor-buyer supply chain model with stochastic lead time. Computers and Operations Research, 2016, vol. 72, pp. 43-49. DOI: 10.1016/j.cor.2016.02.002.

\section{Информация об авторах}

Щадов Иван Михайлович - доктор технических наук, профессор, заведующий кафедрой управления промышленными предприятиями, Иркутский национальный исследовательский технический университет, 664074, г. Иркутск, ул. Лермонтова, 83, e-mail: Ivanschadov@inbox.ru.

Конюхов Владилир Юрьевич - кандидат технических наук, профессор, кафедра управления промышленными предприятиями, Иркутский национальный исследовательский технический университет, 664074, г. Иркутск, ул. Лермонтова, 83, e-mail: kvu@invest38.com.

Челезов Александр Владимирович - директор ПО «Норильскремонт» ООО «Норильскникельремонт», 663302, г. Норильск, пл. Гвардейская, 2, e-mail: chemezovav@gmail.com.

Беляевская Талара Сергеевна - студент магистратуры Института менеджмента инноваций, Национальный исследовательский университет «Высшая школа экономики», 101000, г. Москва, ул. Мясницкая, 20, e-mail: tamara1294@mail.ru.

\section{Authors}

Ivan M. Schadov - Doctor of Technical Sciences, Professor, Head of the Department of Management of Industrial Enterprises, Irkutsk National Research Technical University, 83 Lermontov St., 664074, Irkutsk, Russian Federation; e-mail: Ivanschadov@inbox.ru.

Vladimir Yu. Konyukhov - PhD in Engineering, Professor, Chair of Industrial Enterprise Management, Irkutsk National Research Technical University, 83 Lermontov St., 664074, Irkutsk, Russian Federation; e-mail: kvu@invest38.com.

Alexandr V. Chemezov - Director of PA «Norilskremont», LLC «Norilsknickelremont», 2 Gvardeyskaya Av, 663318, Norilsk, Russian Federation; e-mail: chemezovav@gmail.com.

Tamara S. Beliaevskaia - Master Degree Student, Institute of Innovation Management, Higher School of Economics, National Research University, 20 Myasnitskaya St., 101000, Moscow, Russian Federation; e-mail: tamara1294@mail.ru.

\section{Библиографическое описание статьи}

Щадов И. М. Инвестирование импортозамещения на предприятиях металлургической отрасли / И. М. Щадов, В. Ю. Конюхов, А. В. Чемезов, Т. С. Беляевская // Baikal Research Journal. — 2016. - T. 7, № 6. — DOI: 10.17150/2411-6262.2016.7(6).5.

\section{Reference to article}

Schadov I. M., Konukhov V. Yu., Chemezov A. V., Beliaevskaia T. S. Investing import substitution at metallurgic enterprises. Baikal Research Journal, 2016, vol. 7, no. 6. DOI : 10.17150/2411-6262.2016.7(6).5. (In Russian).

\section{Baikal Research Journal}

